Revue des patrimoines

33 | 2017

Patrimoine culturel immatériel et institutions patrimoniales

\title{
Curateur ou traducteur? Exposer le patrimoine culturel immatériel en Alsace Bossue
}

Curator or translator? Exhibiting the intangible cultural heritage in the Alsace Bossue region

\section{Noël Barbe et Jean-Christophe Sevin}

\section{(2) OpenEdition}

Journals

Édition électronique

URL : http://journals.openedition.org/insitu/15596

DOI : 10.4000/insitu. 15596

ISSN : $1630-7305$

Éditeur

Ministère de la culture

Référence électronique

Noël Barbe et Jean-Christophe Sevin, «Curateur ou traducteur ? Exposer le patrimoine culturel immatériel en Alsace Bossue », In Situ [En ligne], 33 | 2017, mis en ligne le 03 novembre 2017, consulté le 19 avril 2019. URL : http://journals.openedition.org/insitu/15596 ; DOI : 10.4000/insitu.15596

Ce document a été généré automatiquement le 19 avril 2019

\section{c) $(9)$}

In Situ Revues des patrimoines est mis à disposition selon les termes de la licence Creative Commons Attribution - Pas d'Utilisation Commerciale - Pas de Modification 4.0 International. 


\section{Curateur ou traducteur? Exposer le patrimoine culturel immatériel en Alsace Bossue}

Curator or translator? Exhibiting the intangible cultural heritage in the Alsace Bossue region

Noël Barbe et Jean-Christophe Sevin

1 «Exposez votre patrimoine culturel immatériel en Alsace bossue » est le titre d'une exposition réalisée en 2014 et itinérante en trois lieux: la Grange aux paysages à Lorentzen (Bas-Rhin), le centre social de Sarre-Union (Bas-Rhin) et le Centre d'interprétation du patrimoine archéologique de Dehlingen (Bas-Rhin).

2 Cette opération patrimoniale, faisant travailler la catégorie du patrimoine culturel immatériel (PCI), a été conduite dans le cadre du parc naturel régional des Vosges du Nord (PNRVN). D'un point de vue principiel - juridique et politique -, elle s'est appuyée tout à la fois sur la convention de l'Unesco pour la Sauvegarde du patrimoine culturel immatériel - s'agissant en particulier de ses injonctions à la participation - et sur la charte du PNRVN nouvellement approuvée ${ }^{1}$. Celle-ci ${ }^{2}$ ne contient pas moins de vingt occurrences du mot immatériel, mis en relation avec celui de patrimoine, qu'il s'agisse de formes souhaitables de son objectivation - «Définir et co-construire un corpus ou une typologie du patrimoine culturel immatériel présent sur l'ensemble du territoire, pour le protéger et le partager ${ }^{3} »-$, de son inscription dans une politique du temps - le préserver "pour garantir aux générations futures l'accès à (sa) connaissance ${ }^{4}$ »-, de son usage potentiel dans une politique d'appropriation du territoire par une capitalisation des données ${ }^{5}$, et enfin de sa valorisation économique lorsqu'il s'agit de lui aménager une place dans le cadre de l'invention d'un "tourisme spécifique Vosges du Nord», en particulier avec la volonté de développer "l'événementiel», soit la promotion d'une forme possible de son effectuation ${ }^{6}$.

3 Tout comme la catégorie de "participation" est employée dans la convention de l'Unesco, celle de «co-construction» l'est dans la charte. Quelle que soit la distance que 
l'on peut prendre avec ces modalités de mise en forme de l'action politique devenues instruments de l'action publique ${ }^{7}$, parfois servant à habiller des opérations de domination, le projet était bien de " faire contribuer ${ }^{8}$ » des habitants d'Alsace bossue ${ }^{9}$ à la définition de la chose patrimoniale. C'est-à-dire de les mettre en situation de faire advenir le patrimoine culturel immatériel à une réalité via le média d'une matérialité et de son exposition. Dans ce processus d'action patrimoniale, l'exposition n'était pas une fin mais un moment.

Il s'agit ici de faire un bref retour descriptif et analytique ${ }^{10}$ sur cette opération, sa mise en place, les modalités de mobilisation des habitants, ainsi que les usages pluriels de la notion de PCI lorsqu'elle est appropriée par des acteurs qui se trouvent mis à l'épreuve parce que sollicités - de s'en saisir pour désigner ou qualifier des biens, des objets, des pratiques... Par appropriation ou usages, nous entendons ceux que la convention de l'Unesco semble autoriser et qui peuvent s'en réclamer, ceux que les experts scientifiques et patrimoniaux promeuvent dans la construction de politiques spécifiques comme ceux qui, bien que revendiqués, ne sont pas autorisés au regard des experts, par exemple parce que ne rentrant pas dans le cadre des empiries du patrimoine culturel immatériel telles que définies par la convention de l'Unesco à l'article 2.

Procès en légitimité peut être fait à ces appropriations dites « ordinaires ", par le monde de l'expertise patrimoniale, bien sûr, mais aussi de leur propre point de vue lorsque, en position de doute - voire de domination -, elles requièrent l'approbation savante. C'est plus précisément sur ces usages faits par des non-experts que portera notre analyse, laissant ici et provisoirement de côté la muséologie - langages, registres, etc.

\section{Mobiliser}

6 Au début était un plan : demander aux habitants de dire ce qu'est, pour eux, le patrimoine culturel immatériel de l'Alsace bossue et le faire en proposant un objet pour une exposition temporaire et itinérante ${ }^{11}$. Pour cela, des opérations de publicisation ont été réalisées dans la presse locale, dans le journal du Parc, etc. Un dépliant a été distribué, qui donnait la définition onusienne du PCI. Il comportait un coupon pour répondre, indiquait téléphone et adresse électronique. Précision était faite que toutes les réponses étaient recevables (fig. 1). 


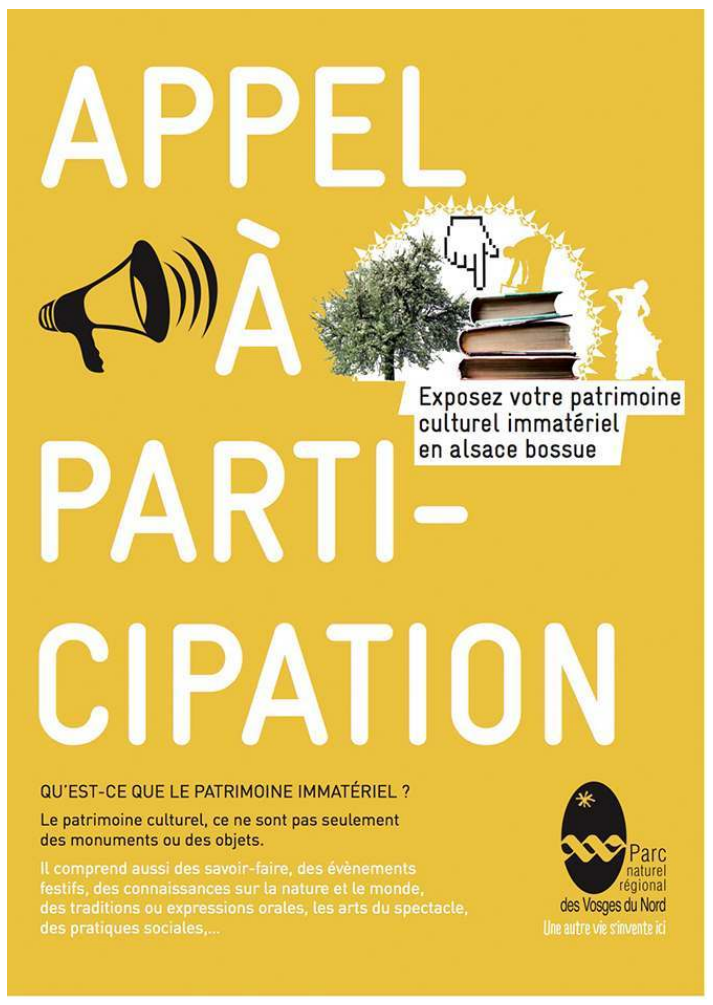

Flyer d'appel à participation.

Le nombre de réponses spontanées s'est révélé très faible. Question de méthode? Réticence des habitants à dire ce qu'ils sont? Le peu d'habitude à donner son avis sur un tel sujet? L'incompréhension de ce qu'est le patrimoine culturel immatériel ? La peur de ne pas être compétent pour répondre? Le fait de ne pas être natif d'Alsace Bossue? L'ensemble de ces raisons a été évoqué, soit des motifs qui renvoient tout à la fois à des questions d'autochtonie et de l'autorisation de la parole qu'elle peut produire tout comme son interdiction mais aussi à des postures de défection face à ce qui peut être perçu comme une expertise institutionnelle supplémentaire ${ }^{12}$. Cela relève aussi des difficultés de traduction et d'appropriation de la catégorie de PCI, et d'un défaut de leur prise en compte dans l'ingénierie du processus de mobilisation des habitants.

Toujours est-il que le plan a dû être modifié... Nous avons alors provoqué des rencontres pour nous faire dire le patrimoine culturel immatériel d'Alsace Bossue, puis suivi des pistes au fur et à mesure qu'elles nous étaient indiquées ; elles pouvaient consister en des personnes mais aussi en des pratiques présentes ou passées. Bref, pour pallier la quasiabsence de réponses spontanées, c'est un ensemble de personnes qu'il a fallu constituer pour faire apparaître des propositions et constituer un public d'enquête ${ }^{13}$. On pourra considérer que cette méthode comporte un biais évident, encore faut-il s'entendre sur celui-ci. Le risque ne réside pas tant dans la non-représentativité ${ }^{14}$ des propositions résultantes que dans le fait de se faire prendre dans des réseaux constitués de personnes pas si non expertes que cela, risque auquel nous avons semblé échapper. Cette enquête en forme de mobilisation de propositions a produit une vingtaine d'objets et autant de rencontres-entretiens avec les prêteurs. Ces objets sont divers, faits de papier ou de métal, de bois ou de peinture, de terre cuite ou de matière plastique ou encore de matière 
académique... Certains ont une valeur marchande quand d'autres, comme de simples photocopies, n'en ont aucune. Ils peuvent être aujourd'hui sans usage ou au contraire être usuellement activés. Mais tous sont constitués par les mots prononcés à leur propos qui agencent du temps, des objets et des hommes. Dans ce glissement vers la parole, ce n'est pas de souvenirs dont il est question, pas plus que de collecte, mais de l'attribution de qualités patrimoniales. Ce sont là différentes manières de définir ce qu'est la chose patrimoniale.

Il s'agissait donc de laisser aller les proposants là où ils le souhaitaient, d'ouvrir à ce que produit la demande et à ce qu'elle fait faire. Dit autrement, il n'était pas question de documenter des objets déjà là mais d'ouvrir un espace d'énonciation où des objets viennent représenter des conceptions de ce qui fait patrimoine. Ce renversement par rapport à une démarche qui aurait été qualifiée par le dispositif de la collecte implique aussi un déplacement de posture. À celle du curateur et de l'expert validant ou non un choix et une propriété patrimoniale des objets, nous avons substitué celle du médiateur ou du traducteur analytique de conceptions patrimoniales non savantes. Dans ce dispositif qui articule savoir et pouvoir ${ }^{15}$, ce n'est pas tant in fine les modalités d'élaboration d'un savoir sur des valeurs qui font écart mais plutôt la mise en public, par l'exposition, de ces conceptions.

\section{Traduire}

10 Dans ces conceptions de la chose patrimoniale énoncées par les propriétaires des objets prêtés, quatre topos, pour lesquels nous donnerons des exemples, peuvent être isolés et mis en avant.

\section{«Le patrimoine, ce sont les relations sociales"}

11 Le patrimoine immatériel peut être compris comme la somme des relations sociales entretenues avec d'autres êtres humains. L'objet matériel est alors ce qui exprime, permet, résulte ou crée ces relations. Il en est ainsi par exemple de la fabrication de pâtes fourrées de farce de viande - les Knepfles - pour laquelle la Wurstmaschine (machine à fabriquer la farce) est centrale. Mais ce qui importe à travers cet objet, c'est le temps partagé de la fabrication. Faire ensemble des Knepfles est l'occasion de réactiver des relations sociales et familiales. Dans cette optique, les usages de la Wurstmaschine et du téléphone portable peuvent coexister en permettant dorénavant que cette préparation des Knepfles soit partagée à distance grâce à l'échange de MMS qui attestent les étapes de la préparation en cours et parfois lui apportent des conseils... (fig. 2) 


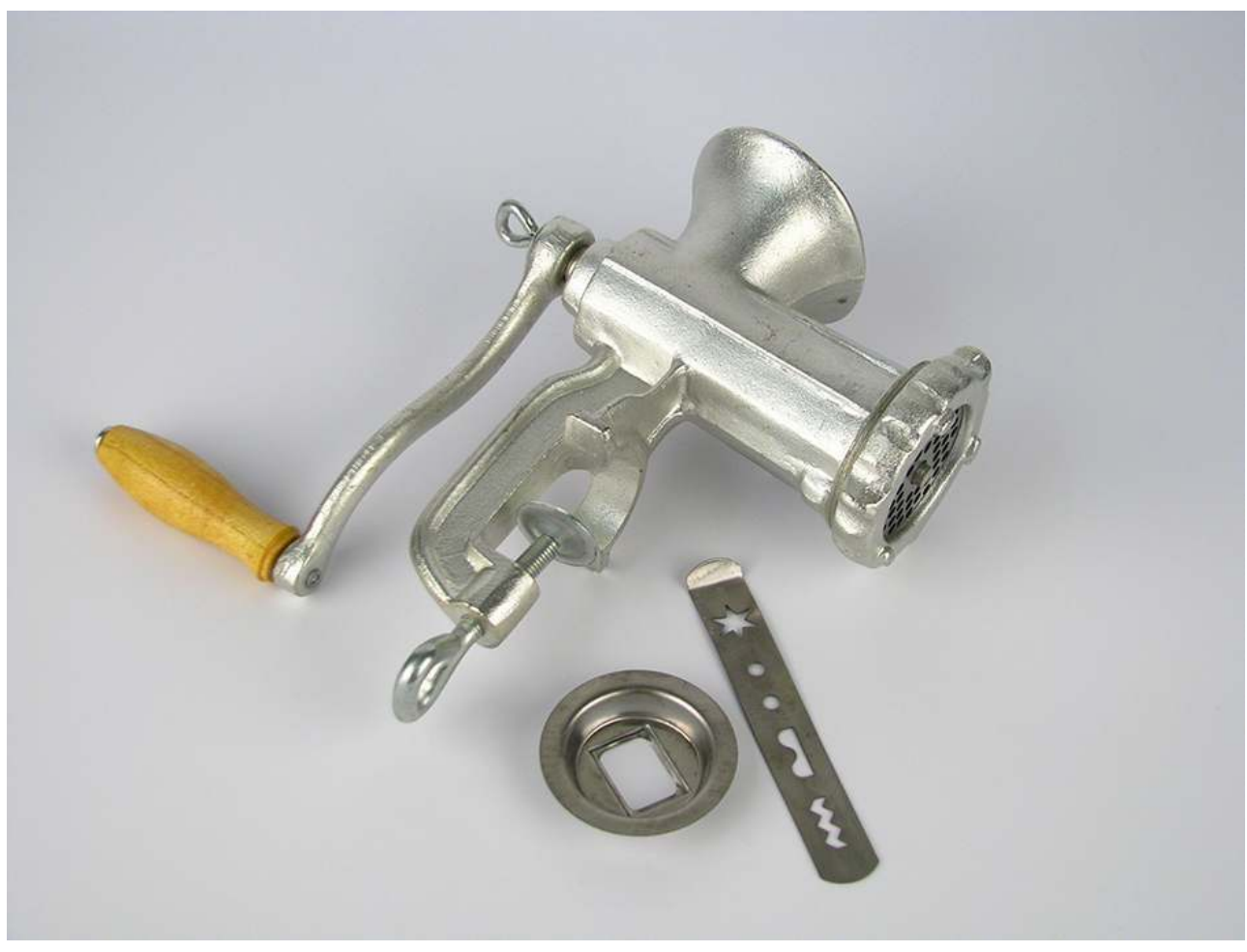

«C'est la collectivité qui prépare ». Wurstmaschine.

Phot. Lætitia Velten, 2016. ( ) Sycoparc/L. Velten.

\section{« Le patrimoine, c'est un héritage actif »}

Poursuivre, actualiser ou revitaliser... Faire du patrimoine un héritage actif consiste ici à se constituer comme le destinataire et le continuateur d'un passé, de ses pratiques et de ses objets. Il peut s'agir de désigner cet héritage attendant son légataire, ou bien de faire vivre une pratique comme le bricolage. Ainsi, la permanence d'une ingéniosité du recyclage peut être décelée dans la pratique du tuning comprise comme un prolongement de manières d'être du monde agricole $\mathrm{du} \mathrm{xx}^{\mathrm{e}}$ siècle, dans une société transformée par l'industrialisation. L'image des longues files de véhicules agricoles livrant à Sarre-Union des pommes pour en faire du jus est aussi sans cesse revenue (fig. 3). Produire des pommes, c'est aussi façonner des paysages, ces paysages vallonnés et ponctués de vergers ceinturant les villages. Continuer à le faire et encourager la production permet de contribuer à leur préservation (fig. 4). 
Figure 3

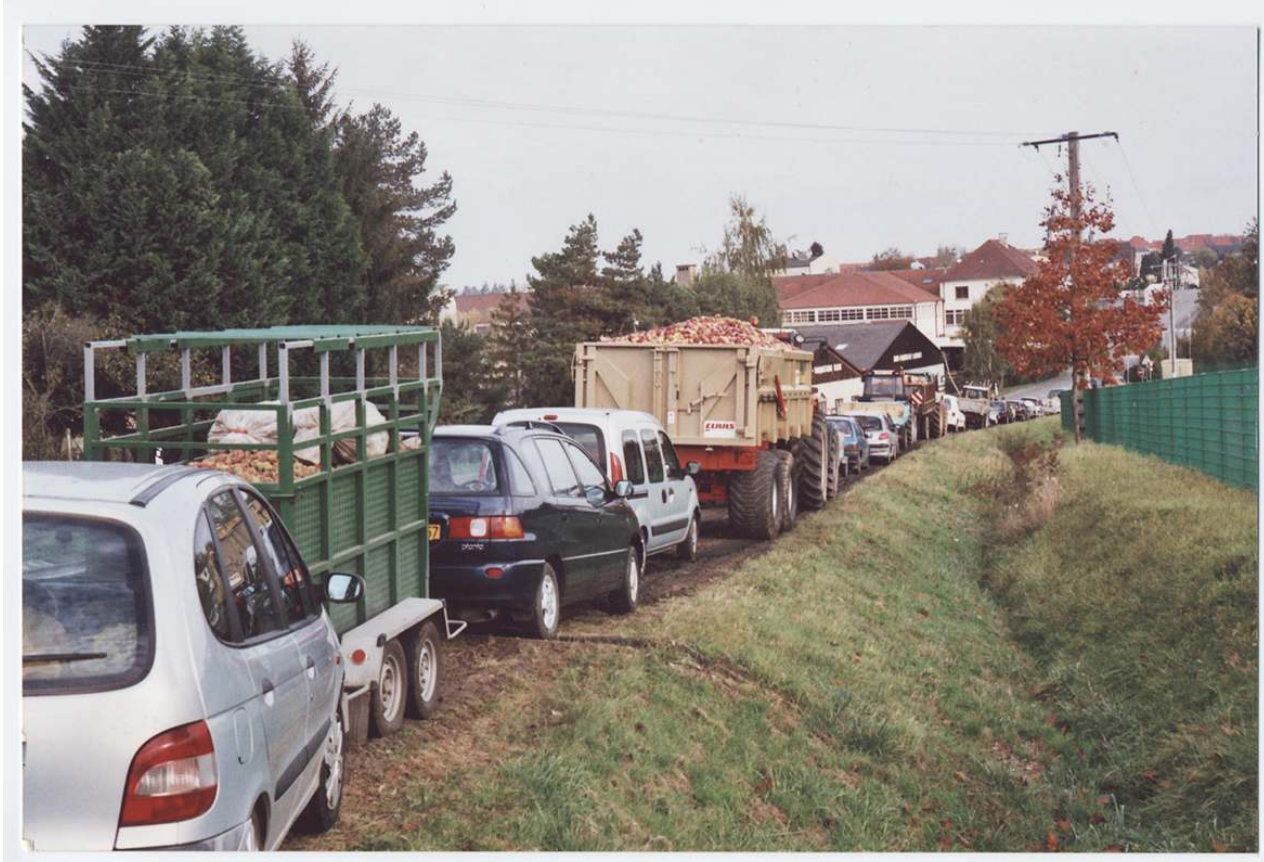

Clichés de livraisons de pommes à Sarre-Union. Collection particulière.

\section{Figure 4}

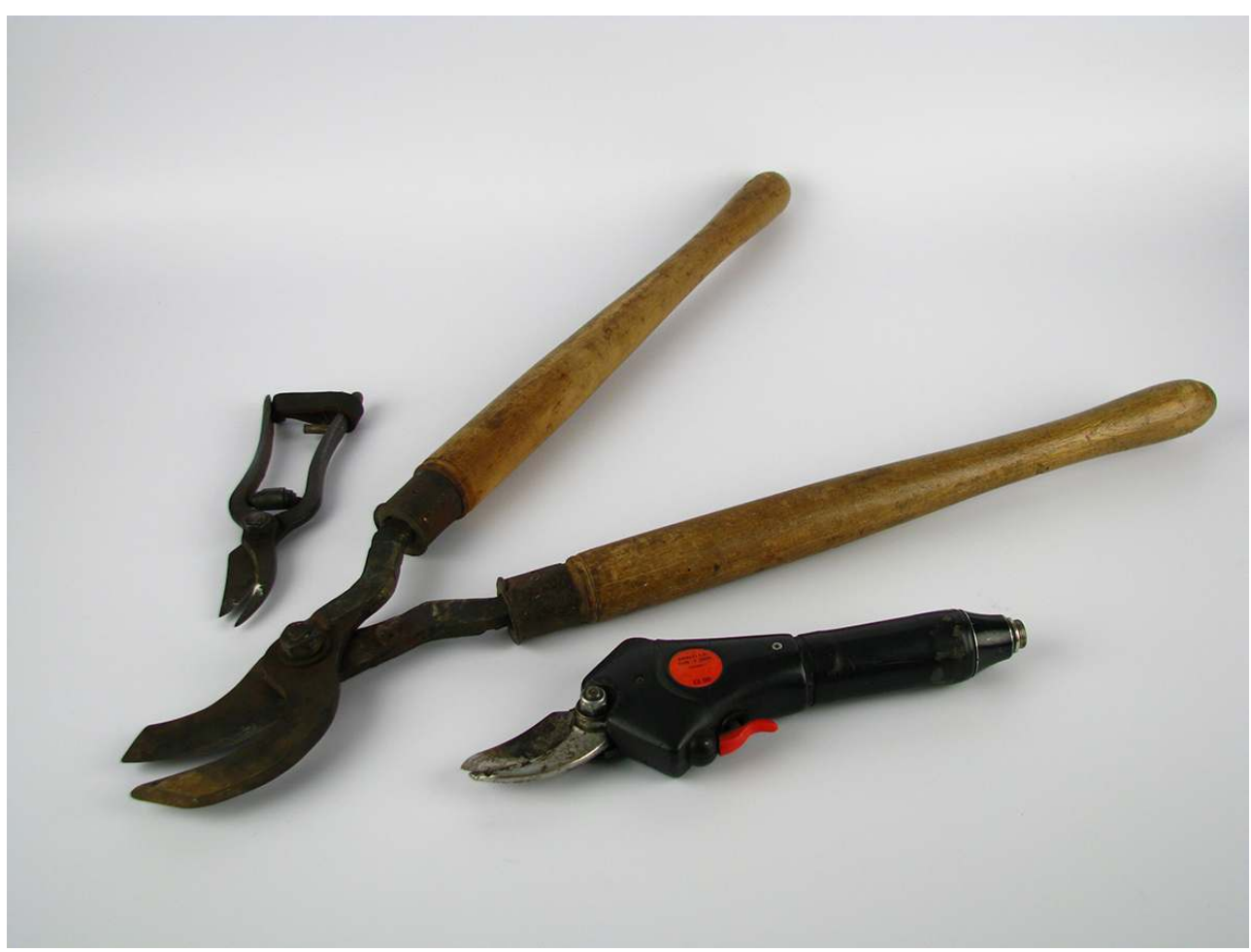

"On ne peut pas seulement avoir des bosses sans aucun arbre pour maintenir une ceinture verte autour du village ». Outils de taille pour les pommiers.

Phot. Lætitia Velten, 2016. ( S Sycoparc/L. Velten. 


\section{« Le patrimoine, c'est une trace»}

13 Le patrimoine comme trace, c'est un regard d'aujourd'hui sur un événement passé que l'on se remémore - un " après-avec » pour reprendre le mot de Jacques Derrida ${ }^{16}$-, mais aussi ce que l'on produit aujourd'hui, sans toujours le savoir, comme trace pour le futur. Quelque chose atteste qu'un fait a eu lieu, que telle chose a été pensée ou pratiquée, et la trace contribue à les documenter. Cela peut être un objet auparavant utilisé, un support papier ou numérique mais aussi un symbole graphique qui semble traverser le temps, comme le motif de la rosace que l'on repère sur une stèle romaine, des encadrements de porte et des tuiles d'Alsace Bossue (fig. 5). Ce symbole graphique est questionné du point de vue d'une éventuelle permanence symbolique dont il serait le témoin. Enregistrer des images d'événements contemporains, les graver sur des supports numériques que l'on classe soigneusement selon leurs sujets, peut être pensé comme la production d'archives pour le futur (fig. 6).

Figure 5

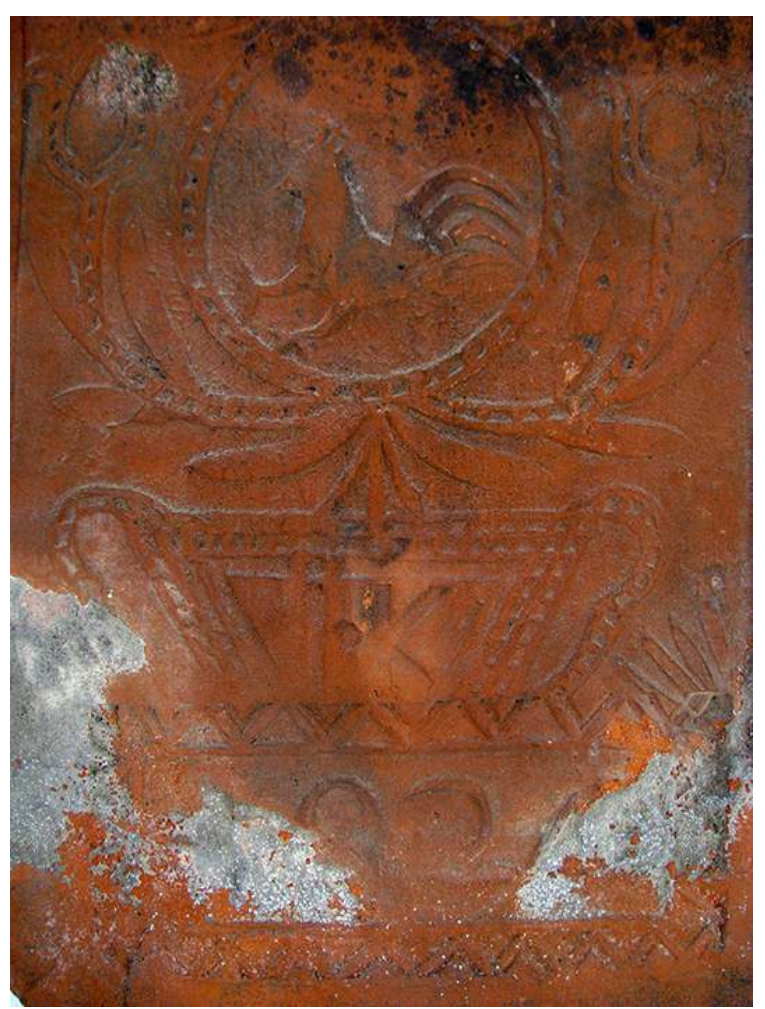

«Les symboles traversent les siècles ». Détail d'une tuile, Ratzwiller.

Phot. Lætitia Velten, 2016. (c) Sycoparc/L. Velten. 


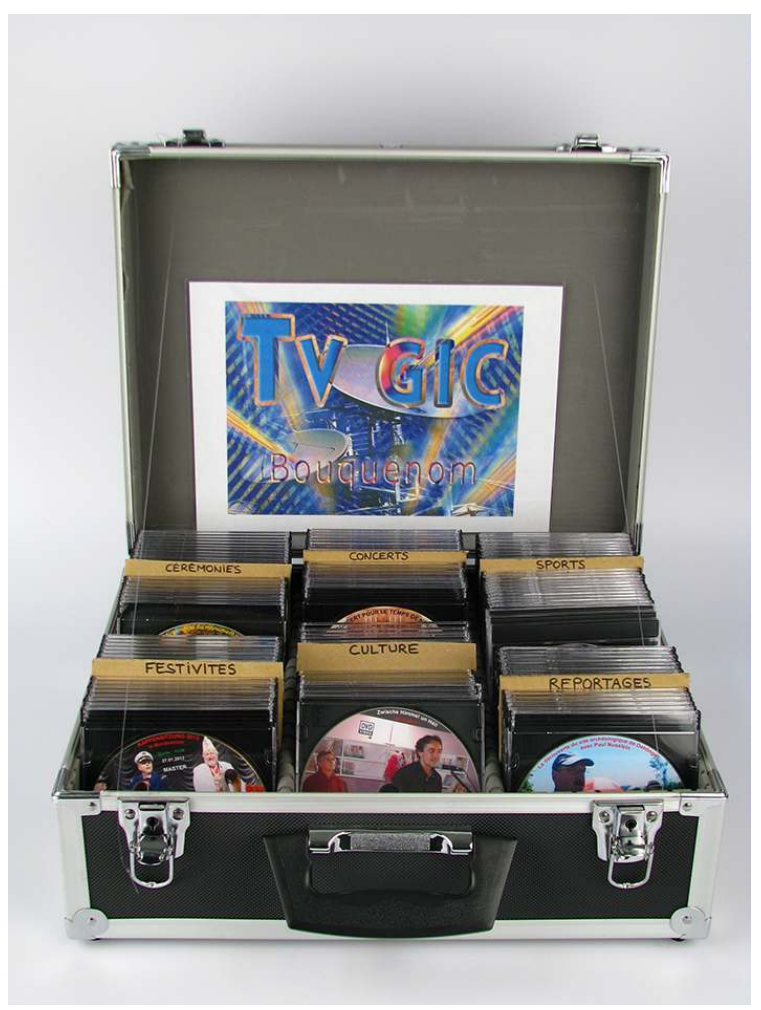

"C'est comme des trésors qui dorment ». DVD classés dans leur valise. Phot. Lætitia Velten, 2016. (c) Sycoparc/L. Velten.

\section{« Le patrimoine, c'est un monde perdu »}

14 Le patrimoine peut être pensé comme ce qui n'est plus, comme ce qui est définitivement passé. Si ce passé existe encore, ce n'est plus que sous la forme de souvenirs ou d'une mémoire pensée comme sans efficacité sur le présent. Ce sentiment de perte peut s'accompagner de nostalgie ou de regrets, comme du constat du temps qui passe et que des choses changent inéluctablement. La Kirb - la fête patronale - contient toutes ces nuances; c'est la perte d'un moment festif et à forte intensité sociale du village, moins importante dorénavant non seulement par le nombre de personnes rassemblées - parce que centrée sur la famille proche et non plus étendue - mais aussi parce que le bal ou le cortège des conscrits ont disparu ou sont d'une moindre ampleur. Mais plus fondamentalement, parce que la valeur des repas, des plats confectionnés et des habits portés spécialement pour cette occasion a changé. Ces pratiques ont été banalisées par l'émergence d'une économie d'abondance, effaçant le caractère extraordinaire de la Kirb elle-même (fig. 7). Cette perte d'un monde se redouble parfois de celle de ses traces, photographies et papiers divers... Une telle disparition a pu se produire durant l'une ou l'autre des situations de violence, comme les guerres et les occupations armées, qu'a traversées cette région. Elles ont pu conduire à une perte totale de biens mobiliers. Dire ou redire ces moments-là, c'est rappeler et se mesurer encore à la violence d'hommes faite à d'autres hommes (fig. 8). 
Figure 7
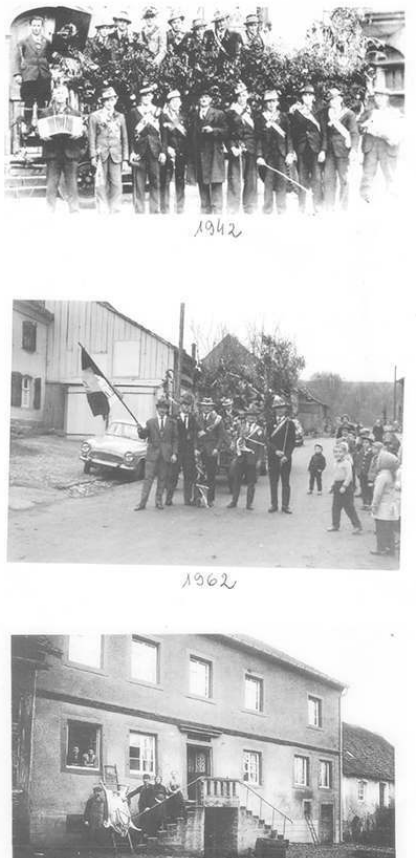

Photocopie de clichés de la Kirb, Delhingen. Collection particulière. 


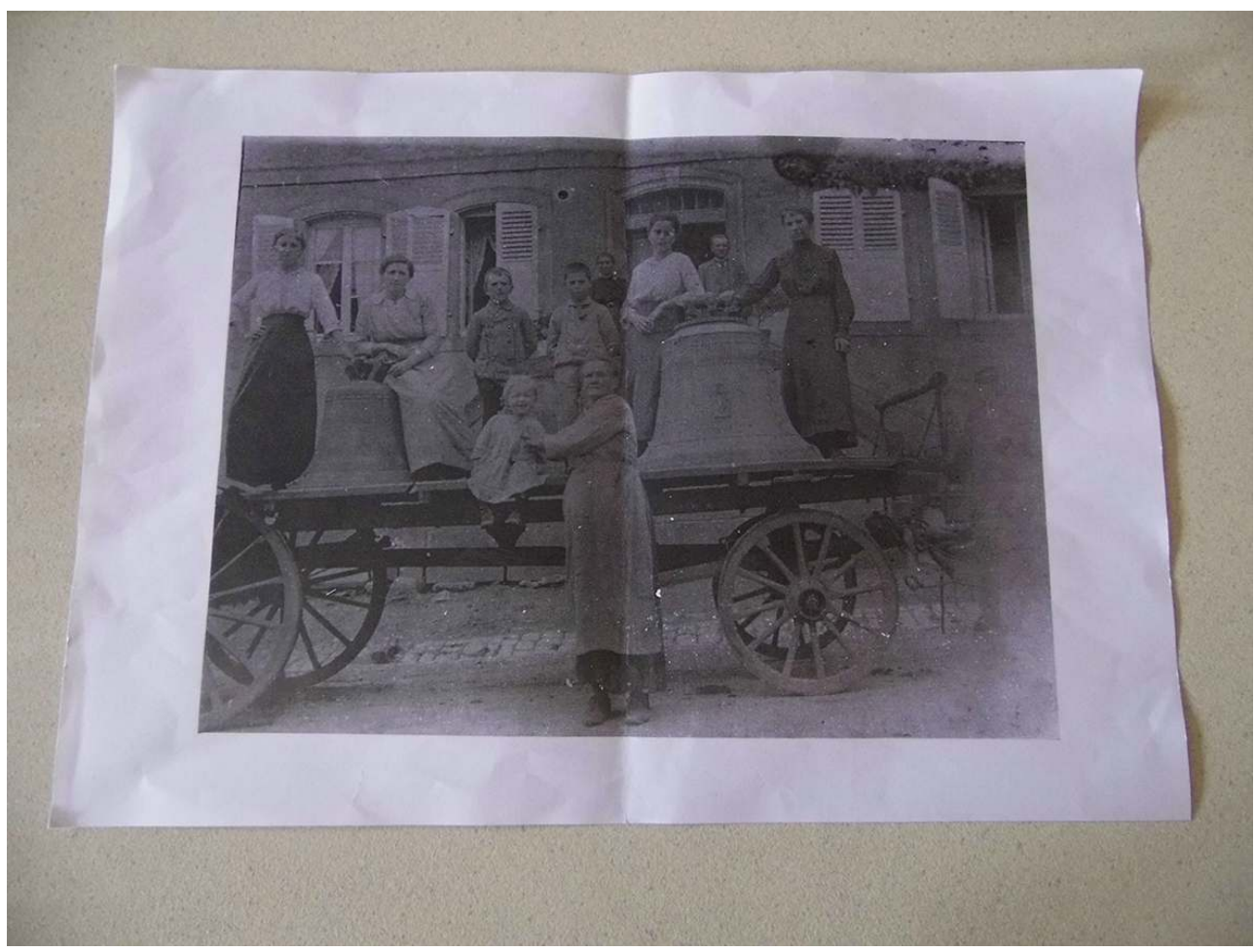

«C'est tout ce que j'ai. J'avais beaucoup de choses et puis on nous a tout volé. On a été expulsé ». Photocopie d'un cliché de la confiscation des cloches par les Allemands à Harskirchen en 1917.

Phot. Noël Barbe, juillet 2014. ( Noël Barbe.

\section{De la parole à la voix ?}

15 L'exposition, structurée autour de ces quatre topos, a été pensée pour fonctionner comme un opérateur d'une tentative d'aménagement du rapport social et politique que constitue le patrimoine. Ces propositions spontanées ou sollicitées peuvent être en effet lues dans l'optique d'une politique du PCI. Il y a d'abord l'ouverture d'un espace de parole autour de la question du patrimoine et de ses conceptions. Cet espace vient déborder la convention en contestant non seulement la division entre matériel et immatériel pour l'articuler selon différentes modalités mais aussi sa définition du point de vue des domaines empiriques qu'elle suppose. Cela produit une distorsion de la catégorie PCI telle qu'elle avait été conçue par les experts de l'Unesco, qui se manifeste plus particulièrement dans les topos du monde perdu et de la trace. Il y a donc là un dissensus. Une posture pédagogique pourrait conduire à juger cette contestation négativement en considérant que les participants « n'ont rien compris ». Tout autre a été la nôtre, qui a consisté à faire de cette contestation un embrayeur et/ou un outil radiographique.

Cette parole qui se manifeste dans ces propositions n'a pas pour objet de témoigner ou de raconter. Il s'agit plutôt de restituer une expérience et de s'en autoriser pour caractériser ce qui, à une échelle plus générale, fait patrimoine ${ }^{17}$. Autrement dit, point là de nostalgie, ni de la mémoire collective ni de matériau patrimonial. Le PCI peut avoir une dimension de subjectivation. 
Il reste la question du passage de la parole à la voix, c'est-à-dire de la prise en compte de ces paroles dans une politique. Comment peuvent-elles compter pour être constitutives d'un intérêt politique et éviter leur transformation en matériau patrimonial, pour penser en termes de démocratie plutôt que de démocratisation ${ }^{18}$ ? La question est en débat et c'est l'enjeu de la mise en œuvre de la charte du Parc entre deux polarités : l'exercice patrimonial comme technologie de gouvernement ${ }^{19}$ ou comme pratique égalitaire et radicalement démocratique ${ }^{20}$, productrice de savoirs positifs et de capacités (fig. 9).

Figure 9

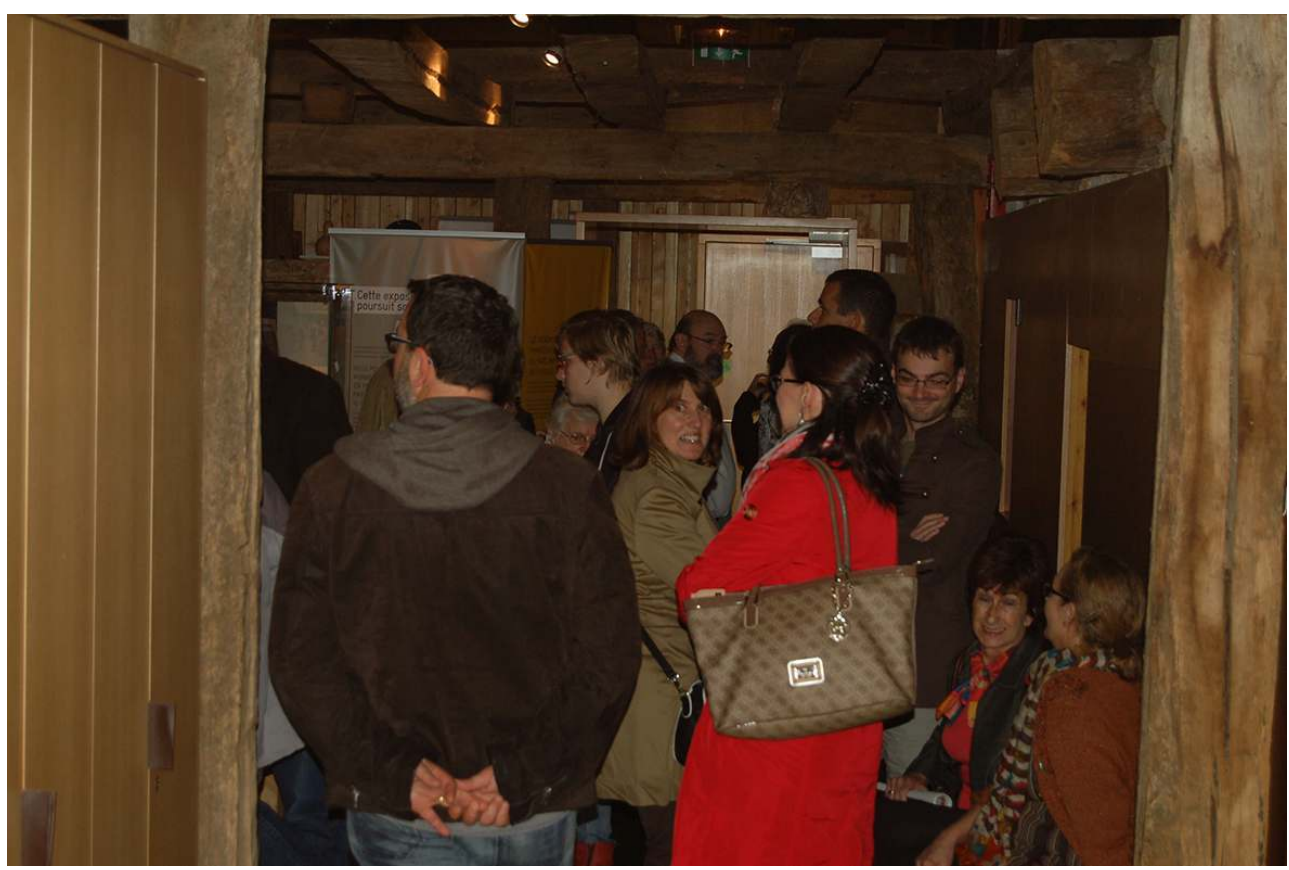

Jour de vernissage, La Grange aux paysages, Lorentzen, 17 octobre 2014.

Phot. Lætitia Velten, 2014. (C) Sycoparc/L. Velten.

\section{Conclusion}

Nous pouvons pour conclure récapituler l'enjeu de cette opération en analysant l'appel émis en direction des habitants au prisme de la notion de participation, telle que Joëlle $Z^{2} \mathrm{sk}^{21}$ en décline les figures possibles et leur portée. Ainsi, l'ouverture envers les propositions, soit envers les habitants laissés libres de s'approprier cette catégorie PCI, peut être considérée comme un débordement d'une relation unilatérale où les propositions seraient sanctionnées par un jugement sur la conformité à une acception stricte du PCI : ce serait la position du curateur ou de l'expert. Mais elle peut être envisagée comme une acceptation de l'interaction entre des habitants et une institution patrimoniale, ce qui correspond à la position du traducteur ou du médiateur que nous avons adoptée. Dans cette perspective, la participation n'apparait pas simplement comme le fait de "prendre part à », en jouant docilement le jeu défini à l'avance par ladite institution, mais bien plutôt comme une contribution ${ }^{22}$. Cette contribution représente une participation active pour laquelle les propositions sont personnelles, tout en marquant un intérêt pour l'espace public et une volonté d'échange. La contribution a ainsi comme caractéristique d'intégrer les contributeurs dans une histoire commune. Et 
l'articulation entre les différentes propositions à une histoire commune aura constitué la part active de notre intervention: ne pas cadrer par avance ou refermer l'espace des possibles tout en maintenant la cohérence de ce cadre en traduisant les propositions, explicitant les enjeux à la lumière des propos des contributeurs et les resituant dans une perspective plus large sur le patrimoine. Au final, on laissera ouverte la question de savoir si la participation aura aussi été une manière d'en retirer une part, un bénéfice pour les porteurs de propositions, qui prendrait notamment la forme d'une reconnaissance ou d'une « encapacitation».

\section{NOTES}

1. - Les parcs naturels régionaux se définissent comme des territoires organisés autour d'un projet de développement durable - terme pour le moins discutable. La charte est le contrat manifestant ce projet, elle est approuvée par les communes, les départements, les régions, les partenaires associatifs et socio-professionnels qui composent le territoire. Elle est révisée tous les 12 ans. S'agissant de celle du PNRVN, voir : Parc naturel régional des Vosges du Nord (PNRVN). Projet de territoire 2025. Charte du parc naturel régional des Vosges du Nord, 2014. Voir sur le site : http://www.parc-vosges-nord.fr/html/telechargement/projet_territoire/

Charte_du_parc_numerique_2014/

Charte_et_plan/1_Rapport_charte_PNR_Vosges_du_Nord_finale_03_2014.pdf [consulté le 8 août 2015].

2. - Elle comporte 139 pages.

3. - Ibid., p. 40.

4. - Ibid., p. 36 .

5. - Ibid., p. 55.

6. - Ibid., p. 91.

7. - Voir sur ce sujet LASCOUMES, Pierre et LE GALÈS, Patrick (dir.). Gouverner par les instruments. Paris: Presses de Sciences Po, 2004, et s'agissant du gouvernement pastoral du patrimoine ethnologique, BARBE, Noël. «Isac Chiva, ethnologie et politique patrimoniale ». Terrain, 2013, 60, p. 148-163.

8. - Sur cette question de contribuer, voir ZASK, Joëlle. Participer. Essai sur les formes démocratiques de la participation. Lormont : Le Bord de l'eau, 2011 et BARBE, Noël. « Expertise et participation. Configurer l'action patrimoniale ». Dans BOURA, Frédérique. Une aventure de l'esprit. L'Inventaire général du patrimoine culturel. Lyon : Lieux dits éditions, 2016, p. 145-151.

9. - L'Alsace Bossue est une microrégion située au nord-ouest de l'Alsace.

10. - Pour une analyse plus détaillée, voir BARBE, Noël, SEVIN, Jean-Christophe. L'Alsace Bossue en vingt objets et quelques autres. Courts voyages dans le patrimoine culturel immatériel. Besançon : Éd. du Sekoya, 2016.

11. - Les auteurs de ce texte avaient mené une expérience similaire, en 2003, avant l'apparition de la catégorie de PCI. Voir BARBE, Noël. «Le déploreur de l'utilité, l'expert fraternel et l'inventeur de science détaché. Production de savoir et action culturelle. Prolégomènes... ». Ethnographiques.org, 2007, 12, http://www.ethnographiques.org/2007/Barbe [consulté le 10/08/2015]; BARBE, Noël, DENÊTRE, Émilie, SEVIN, Jean-Christophe. «Une archéologie des patrimoines ». Dans Patrimoines singuliers. Chacun son patrimoine. Journal de l'exposition, Lons-le- 
Saunier, musée d'Archéologie, 5 avril-30 novembre 2003, p. 1 et 17-20, et ce qu'en dit POULOT, Dominique. «Le patrimoine immatériel en France entre renouveau muséographique et territoire de projet ». Ethnologies, 2009, 1, p. 165-200.

12. - La défection ne doit pas être entendue comme une attitude qui relèverait simplement de la passivité ou de l'indifférence, voir HIRSCHMAN, Albert O. Défection et prise de parole. Théorie et applications. Paris: Fayard, 1995. Elle peut être volonté d'échapper aux mesures, incitations, injonctions et s'appuyer sur d'autres façons de penser le politique. Voir également TOUAM BONA, Dénètem. Fugitif, où cours-tu ? Paris : PUF, 2016.

13. - Au sens de DEWEY, John. Le Public et ses problèmes. Paris : Gallimard, 2010.

14. - Tarte à la crème du positivisme qui, dans ce cas, supposerait que le PCI préexistait au travail que nous avons mené, dont l'objectif alors n'aurait été que de reconnaître ce qui était déjà là.

15. - FOUCAULT, Michel. «Qu'est-ce que la critique? ». Bulletin de la Société française de philosophie, 2001, n 2, p. 35-63, et « Pouvoir et savoir ». Dans Id. Dits et Écrits II. 1976-1988. Éd. Daniel Defert et François Ewald. Paris : Gallimard, 2001, p. 399-414.

16. - DERRIDA, Jacques. Spectres de Marx. L'État de la dette, le travail du deuil et la nouvelle Internationale. Paris : Galilée, 1993.

17. - Cette question est centrale en France dans des dispositifs de participation et de délibération où les individus sont sollicités en tant qu'habitants dont l'horizon délibératif est maintenu à une échelle locale. Voir BLONDIAUX, Loïc. « Où en est la démocratie participative locale en France? Le risque du vide ». Les Cahiers $d u$ DSU, septembre 2002, 35, p. 9-10 ; BLONDIAUX, Loïc, SINTOMER, Yves. "L'impératif délibératif». Rue Descartes, 63, 2009, p. 28-38, et NEVEU, Catherine. «Habitants, citoyens : interroger les catégories ». Dans BACQUÉ, Marie-Hélène et SINTOMER, Yves (dir.). La Démocratie participative. Histoire et généalogie. Paris : La Découverte, 2011, p. 39-50.

18. - Démocratisation qui repose sur des soubassements aristocratiques comme l'est la procédure du vote aux $\mathrm{XIX}^{\mathrm{e}}$ et $\mathrm{XX}^{\mathrm{e}}$ siècles. Plus de personnes sont touchées par le patrimoine, mais ont-elles leur mot à dire sur ce qui fait à leurs yeux patrimoine. Un parallèle peut être dressé avec les mécanismes du gouvernement représentatif moderne. Voir à ce propos VAN REYBROUCK, David. «Cher président Juncker». Dans GEISELBERGER, Heinrich (dir.). L’Âge de la régression. Paris: Premier Parallèle, 2017, p. 277-295.

19. - BROSSAT, Alain. Le Grand dégoût culturel. Paris : Éd. du Seuil, 2008.

20. - Dans le sens agonistique, voir MOUFFE, Chantal. Agonistique. Penser politiquement le monde. Paris : Beaux-Arts de Paris éd., 2014.

21. - ZASK, Joëlle. Participer : essai sur les formes démocratiques de la participation. Op. cit. (2011).

22. - Voir BARBE, Noël. «Expériences patrimoniales, valeur sociale ou portée politique?». Culture et recherche, 2016, $\mathrm{n}^{\circ} 133$, p. 60-62.

\section{RÉSUMÉS}

À partir d'un retour descriptif et analytique sur une opération patrimoniale mettant au travail la catégorie de patrimoine culturel immatériel, ce texte en expose les enjeux en termes de démocratie patrimoniale. Avec l'exposition intitulée «Exposez votre patrimoine culturel immatériel en Alsace Bossue ", réalisée en 2014, le projet était de faire contribuer des habitants de cette microrégion d'Alsace à la définition de la chose patrimoniale. Exposer n'était pas tant une fin qu'un moment du processus puisqu'il s'agissait de mettre les habitants en situation de 
faire advenir le patrimoine culturel immatériel à une réalité via le média d'une matérialité proposée et de sa présentation argumentée ou documentée dans l'espace public, i.e. de porter l'attention sur la territorialisation de cette catégorie, les manières de la définir et les débordements de l'expertise en la matière, les propositions d'articulation entre le matériel et l'immatériel.

Based on a description and analysis of the exhibition project Exposez votre patrimoine culturel immatériel en Alsace Bossue ('Exhibit your Intangible Cultural Heritage in the Alsace Bossue region'), this text examines the issues that were at stake in this project terms of heritage democracy. The guiding concept of the exhibition that was organised in 2014, was to transform inhabitants into participants, making their contribution to the definition of the heritage of this region in Alsace. In this sense the exhibition was above all a moment in an on-going process. The main aim was to give intangible cultural heritage (ICH) a measure of reality using the medium of material objects offered by local inhabitants, then drawing attention to the way these inhabitants define the ICH, and how this coincides with the official appraisal of heritage institutions.

\section{INDEX}

Mots-clés : patrimoine culturel immatériel, démocratie patrimoniale, épistémologie politique du patrimoine, exposition, sciences sociales, politisation, parcs naturels régionaux

Keywords : intangible cultural heritage, democratic practices and heritage, political epistemology and heritage, exhibition, social sciences, politicisation of heritage, regional natural parks

\section{AUTEURS}

\section{NOËL BARBE}

Chercheur à l'Institut interdisciplinaire d'anthropologie du contemporain et conseiller pour l'ethnologie au ministère de la Culture (DRAC Bourgogne Franche-Comté) noel.barbe@cnrs.fr

\section{JEAN-CHRISTOPHE SEVIN}

Maître de conférences, Équipe Culture et Communication du Centre Norbert-Elias, Université d'Avignon jean-christophe.sevin@univ-avignon.fr 